A N N A L E S Annales de Bretagne et des Pays de l'Ouest

\title{
Du Guesclin - Images et histoire
}

\section{Michael Jones}

\section{(2) OpenEdition}

Journals

Édition électronique

URL : http://journals.openedition.org/abpo/3339

DOI : $10.4000 /$ abpo.3339

ISBN : 978-2-7535-5185-5

ISSN : 2108-6443

Éditeur

Presses universitaires de Rennes

Édition imprimée

Date de publication : 28 juillet 2016

Pagination : 189-192

ISBN : 978-2-7535-5183-1

ISSN : 0399-0826

Référence électronique

Michael Jones, "Du Guesclin - Images et histoire », Annales de Bretagne et des Pays de l'Ouest [En ligne], 123-2 | 2016, mis en ligne le 28 juin 2016, consulté le 23 septembre 2020. URL : http:// journals.openedition.org/abpo/3339; DOI : https://doi.org/10.4000/abpo.3339 
salle princière de Charles I ${ }^{\mathrm{er}}$ déjà évoquée et les peintures du chœur de la cathédrale récemment retrouvées derrière les lambris. Cette mise en image de la vie de Maurille et de René n'est pas encore bien élucidée et C. Davy propose d'y voir, outre le fait de contribuer à la transformation du chœur en un vaste reliquaire consacré aux deux saints, aussi une adhésion à la politique italienne de Charles $\mathrm{I}^{\mathrm{er}}$.

Le volume se clôt par une brève étude du culte de saint Louis en Anjou, difficile à connaître mais suivi ici à travers les manifestations artistiques qui en subsistent. Si le saint roi fut honoré, l'Anjou ne semble pas s'être distingué particulièrement. On peut ici regretter l'absence d'un grand texte de synthèse qui reprendrait les lignes directrices qui se dégagent de toutes ces études et donnerait une plus grande homogénéité au livre. À vrai dire, le coordinateur E. Vacquet le fait dans une remarquable mais trop courte introduction. Il souligne que l'Anjou a d'abord continué sur son héritage plantagenêt particulièrement brillant. L'arrivée des Capétiens modifie assez peu de choses et c'est avec l'arrivée de Charles d'Anjou que l'évolution semble se produire. Le gothique rayonnant se fait une place, comme le décor héraldique, mais l'art du vitrail comme celui de la sculpture disparaissent faute de commandes et de chantiers. Ce livre témoigne de la diversité et de la richesse des enquêtes menées et leur publication constitue un apport essentiel en les portant à la connaissance du plus grand nombre.

Daniel PICHOT

MoAL, Laurence, Du Guesclin - Images et histoire, Rennes, PUR, 2015, 335 p., $35 €$.

Si l'on excepte les monarques tels que Saint-Louis ou Louis XI, Bertrand du Guesclin est sans aucun doute, après Jeanne d'Arc, la figure médiévale qui en France continue le plus à attiser les passions et susciter l'intérêt des historiens, des écrivains et des artistes ainsi que du grand public. Parmi les biographies récentes, celles de Georges Minois (Du Guesclin, 1993) et Richard Vernier (The Flower of Chivalry. Bertrand du Guesclin and the Hundred Years War, 2003) sont toutes deux agréables à lire et généralement sérieuses; récemment le travail un peu provocateur de Thierry Lassabatère (Du Guesclin. Vie et fabrique d'un héros médiéval, 2015) a apporté une contribution intéressante à l'étude de l'élaboration d'un mythe qui, commencé du vivant de Du Guesclin, se poursuit encore de nos jours, le propulsant à la position prééminente qu'il occupe parmi les figures de la chevalerie de la fin du Moyen Âge. Grâce à une recherche impressionnante et abondamment illustrée sur les différentes façons dont la figure du connétable a été décrite au cours des siècles, Laurence Moal apporte ici une dimension nouvelle et très séduisante à la compréhension de la manière dont l'homme et sa légende ont été appréciés ou interprétés. Elle commence par les enluminures de manuscrits presque contemporains et par les monuments funéraires érigés à sa mémoire, puis en passant par les gravures anciennes, statues, peintures, dessins et autres formes littéraires ou théâtrales, elle nous amène jusqu'aux médias modernes, y compris le cinéma, la télévision et les bandes dessinées. Les reconstitutions historiques, devenues populaires au XIXe siècle, ont aussi attiré un public nombreux, surtout à partir des années 1980. Arguant de ce que peu sinon aucune de ces images innombrables ne sont de simples représentations, $\mathrm{M}^{\mathrm{me}}$ Moal questionne en permanence le raisonnement de leurs auteurs ou de ceux qui les ont détruites, comme c'est arrivé à plusieurs statues de Du Guesclin en raison d'une opposition anachronique et mal renseignée vis-à-vis de ses supposées positions politiques ou des idées et intentions de ceux qui l'employèrent. Le but 
de L. Moal est clairement énoncé : " Les images de Du Guesclin sont à considérer dans leur globalité, en prenant en compte leur sens mais aussi leurs usages, leurs fonctions, leurs différents niveaux de réception dans des contextes sociaux, politiques, idéologiques en constant renouvellement. Comment évolue la perception de Du Guesclin à travers les siècles? Et comment les images produites ont-elles pu conforter ou infléchir cette représentation?" (p. 12).

Le développement qui s'ensuit est divisé en trois parties principales. La première traite de la période des XIv et XV ${ }^{\mathrm{e}}$ siècles. Dans le chapitre I (p. 16-52), nous avons un exposé clair des quatre inhumations séparées des différentes parties du corps du connétable (les viscères au Puy, la peau à Montferrand, le cœur à Dinan et les os à Saint-Denis), de l'impressionnante cérémonie commémorative organisée à Saint-Denis en 1389, de la réalisation du célèbre gisant qui se trouve toujours à Saint-Denis (1397) et des autres monuments élevés à sa mémoire par la suite. Le chapitre II (p. 53-111) comporte une étude approfondie des enluminures de manuscrits médiévaux figurant divers épisodes de la vie de Bertrand, ainsi que de la manière dont son portrait dans la littérature a évolué et comment il a été exploité pour soutenir l'idéologie royale. Il est alors considéré comme le chevalier parfait " qui associe des qualités morales à des qualités guerrières [...]. L'image [...] est un instrument pour mettre en scène l'autorité du prince. L'association avec les images du roi participe à l'édification d'un discours, celui de la prise de possession de la totalité du territoire " (p. 105).

Après l'intérêt considérable suscité par sa vie et ses exploits tout au long $\mathrm{du} \mathrm{Xv}$ e siècle, on constate un déclin relatif au $\mathrm{Xvl}^{\mathrm{e}}$ siècle avant un renouveau au $\mathrm{XVII}^{\mathrm{e}}$ siècle. L'adaptation en prose par Claude Ménard (1618) de la vie en vers de Cuvelier de 1385 (sur laquelle en fin de compte toutes les biographies du connétable sont fondées) est une première étape, mais surtout l'Histoire de Bertrand Du Guesclin... de Paul Hay du Chastelet (1666) est la première biographie moderne sérieuse, surtout utile maintenant pour ses pièces justificatives. Puis, comme $\mathrm{M}^{\text {me }}$ Le Moal le souligne surtout dans la deuxième partie (p. 123-235), à partir du $\mathrm{XVIII}^{\mathrm{e}}$ siècle, le personnage de Bertrand " héros national, défenseur de la nation française " est adopté à la fois par les monarchistes, puis, à partir du milieu du $\mathrm{XIX}^{\mathrm{e}}$ siècle, par les républicains de tout genre avec un extraordinaire enthousiasme. L'analyse de la manière dont Du Guesclin a été présenté comme modèle et héros national dans les manuels scolaires depuis Guizot jusqu'à la veille de la Seconde Guerre mondiale est ici très instructive.

Deux périodes plus courtes mais significatives de l'intérêt qu'il suscita chez les sculpteurs et les pouvoirs publics sont aussi bien étudiées, d'abord au début du XIX ${ }^{\mathrm{e}}$ siècle, puis à nouveau vers 1900 quand plusieurs statues majeures encore en place à Nantes, Dinan et ailleurs furent érigées, tandis que l'une d'entre elles sculptée par Dominique Molknecht en 1825, autrefois au jardin du Thabor, à Rennes est aujourd'hui disparue. Parmi celles érigées en dehors de la Bretagne, celle de Châteauneuf-de-Randon (Lozère) en 1894 est peut-être la plus impressionnante. Transformée en monuments aux morts de la Grande Guerre, elle a reçu une nouvelle plaque en 1980 pour commémorer le $600^{\mathrm{e}}$ anniversaire de la mort du connétable sous sa tente pendant le siège de la ville (c'est peut-être la scène qui revient le plus souvent dans l'iconographie de Bertrand). L'inauguration de tels monuments entraînait habituellement des bombances municipales, fêtes, concerts et représentations théâtrales dont certaines sont décrites de façon très vivante, tandis que les pièces les plus notables comme Messire Du Guesclin de Paul Déroulède (1895) ou NotreDame-Guesclin de Théodore Botrel (1906) sont étudiées en détail. Une conclusion importante en découle : "Ce qui compte, c'est l'image de l'image. Le portrait s'est figé. Du Guesclin n'est plus seulement une légende, il est devenu une caricature " (p. 226). 
Dans la troisième partie (p. 237-313), Laurence Moal montre comment, après 1918, les politiciens, les hommes d'État et les vieux soldats comme le maréchal Foch continuent d'exploiter le héros médiéval qui maintient en grande partie sa popularité en France, bien qu'il soit de plus en plus rejeté par les nationalistes bretons à partir des années 1920. En Bretagne, la supposée traitrise du connétable envers le duché à la fin de sa vie est encore aujourd'hui pour certains un sujet de grand émoi et de colère.

Les nouveaux médias ont aussi offert des opportunités supplémentaires d'exploiter les mythes et légendes qui se sont développés autour de lui. Bertrand a été le sujet de nombreux films, mais aucun n'est considéré par $\mathrm{M}^{\mathrm{me}}$ Moal comme ayant eu beaucoup de retentissement, mis à part celui en noir et blanc dirigé par Bernard de Latour et Pierre Billon, Du Guesclin (1949) dont la réalisation est traitée en détail. Peut-être plus influente auprès du grand public, surtout dans les années 1950-1960, est la représentation de Du Guesclin dans les bandes dessinées (une forme d'art et de littérature populaires qui, mis à part Tintin et Astérix, n'a pas joui d'autant de succès commercial ni d'autant d'attention de la part de chercheurs sérieux dans le monde anglophone qu'il en a eu en France, en Belgique et en Allemagne).

L'auteur de ce compte rendu, britannique, n'est donc pas terriblement qualifié pour porter un jugement sur les nuances de l'analyse de $\mathrm{M}^{\text {me }}$ Moal, que ce soit sur le mérite artistique ou sur les implications politiques et sociales des innombrables images de Du Guesclin que les PUR ont reproduites si remarquablement et si généreusement dans ce livre. C'est en particulier le cas quand nous quittons le Moyen Âge pour des temps beaucoup plus récents où Du Guesclin se retrouve parfois entre les mains de personnes ayant des intentions extrêmement variées, prêtes à transformer une histoire relativement simple en quelque chose de beaucoup plus compliqué pour l'adapter à leur propre fantaisie. Mais il est intéressant de considérer combien l'expérience anglaise est très différente quand il s'agit des héros qui ont fait la nation, comme le reflet d'une vision différente de l'histoire que nos deux grandes nations ont partagée au long des siècles. Il est difficile de trouver un héros anglais du Moyen Âge connu encore au-delà du cercle des universitaires, qui ait fait l'objet, à travers les générations, de différents traitements par des écrivains, des artistes ou même des politiciens et qui joue un rôle équivalent à celui de Du Guesclin dans notre histoire. Les deux personnalités qui s'en rapprochent le plus sont le roi Arthur et Robin des Bois, reconnus maintenant comme mythiques, même s'ils peuvent avoir eu un (ou des) modèle(s) historique(s) sur lequel(s) se sont amalgamés légendes et faits historiques pour créer leur image. À part ces deux figures, les héros médiévaux qui ont survécu dans les mémoires sont tous des rois, à l'exception du Prince noir ( $†$ 1376) : Alfred le Grand ( $†$ 899), Richard I ${ }^{\text {er }}$ Cour de Lion († 1199) et Henry V $(\dagger 1422)$, et un grand méchant, le roi Jean († 1216). Dès la fin de la période anglosaxonne, la monarchie anglaise était déjà fermement installée et le pays largement unifié sous son autorité. En dépit de quelques vacillements (les Anglais au Moyen Âge avaient l'habitude de déposer et de tuer les monarques incapables tout en conservant du respect pour l'institution de la royauté), il en est toujours ainsi, même si le pouvoir royal est maintenant entravé par des limitations constitutionnelles et est devenu largement cérémoniel. Cela contraste avec la France où les destinées de la monarchie, en particulier au temps de Du Guesclin, étaient toujours en jeu et où les intérêts concurrents et les revendications des nombreuses provinces du royaume et de leurs princes ne s'accordaient pas toujours avec ceux du monarque, et où les rois (et après 1789 les régimes républicains) ont eu à se battre presque à chaque génération pour éviter le morcellement et pour promouvoir l'unité nationale en utilisant, comme $\mathrm{M}^{\text {me }}$ Moal l'a amplement démontré ici, des figures comme celle de Du Guesclin afin de renforcer leur message. Cette tradition atteignit son 
apothéose dans la canonisation, aussi récemment qu'en 1920, de Jeanne d'Arc, ce personnage médiéval encore plus iconique. D'où deux trajectoires politiques très différentes : vive la différence!

Michael JONES

Rioult, Jean-Jacques, Toscer-Vogel, Catherine (dir.), Architecture rurale en Bretagne. 50 ans d'inventaire du patrimoine, Lyon, Édition Lieux-Dits, 2014, 324 p., $27 €$.

La Bretagne est sans doute une des régions de France où l'habitat rural non élitaire des $\mathrm{XVI}^{\mathrm{e}}$-XIX ${ }^{\mathrm{e}}$ siècles - ce que l'on appelle fréquemment l'habitat vernaculaire en France depuis les années 1980 - a été le plus étudié ces dernières décennies. À ce titre, l'ouvrage coordonné par Jean-Jacques Rioult et Catherine Toscer-Vogel, tous deux Conservateurs en chef du patrimoine au sein du service de l'Inventaire du patrimoine culturel de la Région Bretagne, s'inscrit dans une déjà longue tradition historiographique.

Cette tradition est tout d'abord celle des inventaires cantonaux dont la publication a été engagée en Bretagne par le Service de l'Inventaire général des monuments et richesses artistiques de la France du Ministère de la Culture (passé en 2004 de la DRAC Bretagne à la Région Bretagne) dès la toute fin des années 1960 et qui intégraient bien évidemment de nombreuses maisons rurales des époques moderne et contemporaine. La publication en 1993 du volume Le manoir en Bretagne, 1380-1600 (C. Mignot et M. Chatenet [dir.], Paris, 1993) apparaissait comme une forme de bilan des inventaires cantonaux pour un type d'habitat rural particulier : les maisons des élites rurales. L'Architecture rurale en Bretagne peut apparaître, à son tour, comme un bilan des inventaires pour les habitats non élitaires. Parallèlement aux travaux du service de l'Inventaire, imprégnés des concepts qui s'étaient développés autour du Musée des Arts et Traditions Populaires puis du Centre d'Ethnologie Française, était publié en 1985, dans la même veine et sous la plume de Daniel Le Couédic et JeanRené Trochet, le volume consacré à la Bretagne de la célèbre collection L'architecture rurale française. Corpus des genres, des types et des variantes, collection publiée entre la fin des années 1970 et le début des années 1990 sous la houlette de Jean CUISENIER, alors justement directeur du Musée des ATP.

Mais l'habitat vernaculaire en Bretagne a fait aussi l'objet de travaux universitaires dès la fin des années 1970 avec la thèse d'un chercheur britannique, bien connu des Bretons, Gwyn Meirion-Jones, thèse soutenue en 1977 et publiée en 1982 (The Vernacular Architecture of Brittany. An Essay in Historical Geography, Edinburg, 1982). Peu après, en 1980, Jean-François Simon soutenait une thèse en Anthropologie sociale et historique à l'EHESS sur les maisons des paysans du Léon (La maison au coeur de l'exploitation agricole traditionnelle en Léon. Le cadre de vie paysan), publiée en 1982 puis suivie, en 1988, d'un volume identique sur la Cornouaille (Tiez. Le paysan breton et sa maison, t. 1 : Le Léon, t. 2 : La Cornouaille, Douarnenez, 1982 et 1988).

Plus récemment, après un relatif désintérêt pour la question dans les années 1990 (exception faite de quelques volumes de l'Inventaire dont le travail d'édition s'est toujours poursuivi), le sujet a de nouveau été traité dans les années 2000-2010 et il convient de citer particulièrement trois publications, originales par rapport aux productions précédentes dans leur approche.

Il s'agit en premier lieu, en 2002, du livre de Jean-Pierre BARDEL et Jean-Luc MAILLARD consacré à un aspect particulier de l'architecture vernaculaire : le bâti en bauge du département de l'Ille-et-Vilaine, technique de construction en terre mas- 